\title{
Characterization of nanolayers at TiAl diffusion bonds
}

\author{
S. Simões*, F. Viana*, A. S. Ramos**, M. T. Vieira ${ }^{* *}$, M. F. Vieira ${ }^{*}$ \\ * CEMUC, Department of Metallurgical and Materials Engineering, University of Porto, R. Dr. Roberto \\ Frias, 4200-465 Porto, Portugal \\ ** CEMUC, Department of Mechanical Engineering, University of Coimbra, R. Luís Reis Santos, 3030-788 \\ Coimbra, Portugal
}

Email: $\underline{\text { ssimoes@fe.up.pt }}$

Aerospace and automotive industries dedicate a special attention to the developments of TiAl alloys, especially to those contributing to the advance toward structural application [1]. Joining technology will be required in nearly all applications of these alloys, processes that balance performance and cost need to be establish. The bonding of these alloys is very difficult due to their high reactivity and tendency to form brittle intermetallic phases. Several techniques are reported in literature to join TiAl [2]. Nevertheless diffusion bonding is the most promising technique as avoids hot cracking, the main problem of fusion welding process [3].

To diffusion bond TiAl alloys with less demanding conditions, the authors use an alternative approach that consist in the deposition of NiAl multilayers on the joining interfaces that modifies the interface, improving the diffusivity and reactivity by promoting nanocrystallinity and increasing the number of interfaces [4].

In this study, the characterization of the TiAl diffusion bonds assisted by NiAl multilayers was performed by scanning transmission electron microscopy (STEM), transmission electron microscopy (TEM), highresolution transmission electron microscopy (HRTEM) and analyzed by energy dispersive X-ray spectroscopy (EDS) and electron energy loss spectroscopy (EELS). NiAl multilayers with a total thickness of $2.5 \mu \mathrm{m}$ and a bilayer thickness of $14 \mathrm{~nm}$ were deposited by dc magnetron sputtering on TiAl surfaces. The bonds were produced at $900{ }^{\circ} \mathrm{C}$ during 60 minutes under a pressure of $5 \mathrm{MPa}$ in vacuum. The diffusion bonding procedure and apparatus is described elsewhere [4].

AlNiTi and $\mathrm{Al}_{2} \mathrm{NiTi}$ intermetallics are identified in an interdiffusion area, Zone 1, and grow perpendicular to the interface as columnar grains, in the diffusion flux direction, with an average grain size of $534 \pm 320$ (Fig. 1 (a) and (b)). At the centre of the interface $\mathrm{Ni}$ and $\mathrm{Al}$ nanolayers transform into B2-NiAl equiaxed grains with an average size of $450 \pm 254 \mathrm{~nm}$. The transition between these two zones, Zone 2, shows nanometric B2-NiAl grains (114 $\pm 76 \mathrm{~nm}$ ). HRTEM images and correspond FFT (Fig. 2) and EELS analyses revealed that nanometric $\mathrm{Ti}$ aligned grains compose the bonding line; these grains are probably the result of $\mathrm{Ti}$ segregation during cooling from the bonding temperature.

This research is sponsored by FEDER funds through the program COMPETE - Programa Operacional Factores de Competitividade - and by national funds through FCT - Fundação para a Ciência e a Tecnologia -, under the project PEst-C/EME/UI0285/2013. 
[1] Dimiduk, D. M., Materials Science and Engineering A, 263, 281-288, 1999.

[2] Froes, F. H. et al., Journal of Material Science, 27, 5113-5140, 1992.

[3] Çam, G. et al., Journal of Material Science, 4, 5273-5282, 2006.

[4] Simões, S. et al., Journal of Material Science, 45, 4351-4357, 2010.

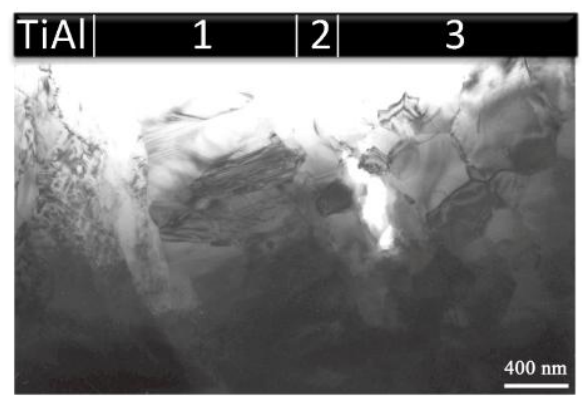

(a)

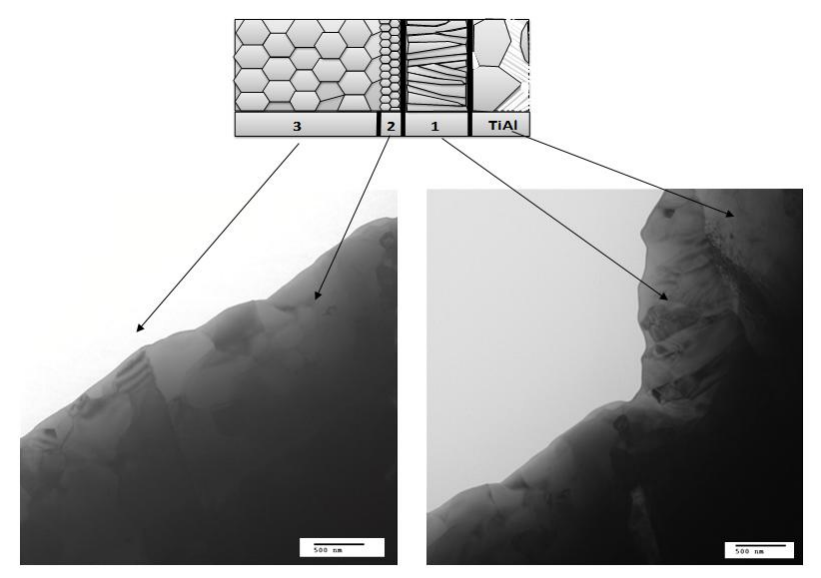

(b)

Figure 1. TEM images of interface obtain at $900{ }^{\circ} \mathrm{C}$ during 60 min under a pressure of $5 \mathrm{MPa}$ with a multilayer period of $14 \mathrm{~nm}$ : (a) low magnification and (b) high magnification of zones 1, 2 and 3.

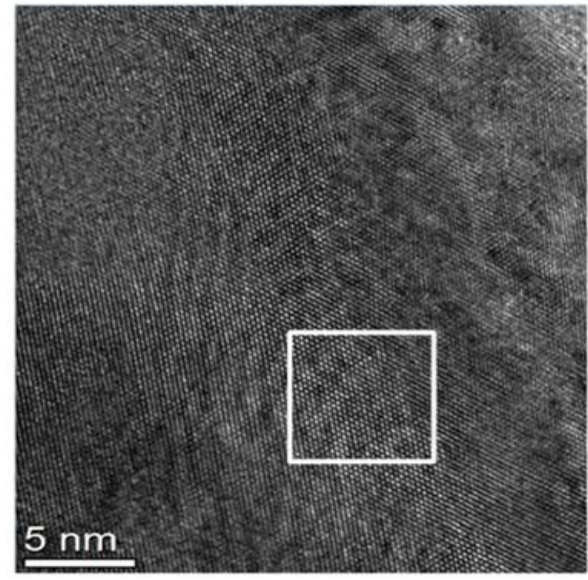

(a)

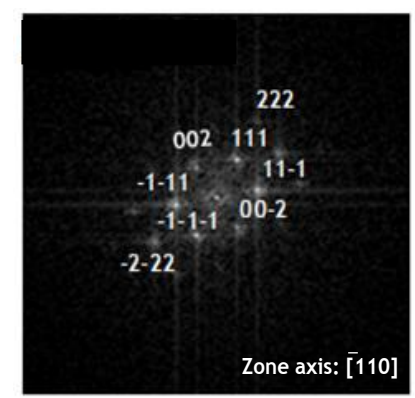

(b)

Figure 2. (a) HRTEM image and (b) FFT of the bonding line showing the presence of titanium grains. 